\section{CHAMACON: exposiciones potencialmente letales en pediatría}

\author{
Bazán-Godínez S ${ }^{1}$, Pérez-Tuñon JG²
}

\begin{abstract}
Resumen
La exposición accidental a sustancias potencialmente tóxicas es un problema frecuente en pediatría. Esta condición puede ser catastrófica dependiendo de factores propios del paciente, su entorno y de la propia sustancia a la que fue expuesto. En este sentido, existe un grupo de sustancias de uso común que al ser ingeridas por un lactante, aun en cantidades mínimas (una tableta, un sorbo o una cucharada), pueden causarle la muerte, ya sea porque son altamente tóxicas o simplemente porque se encuentran en una gran concentración. Cuando un niño ingiere cualquier sustancia de este grupo debe reconocerse como una "exposición potencialmente letal en pediatría". En el presente trabajo se propone la nemotecnia CHAMACON para lograr un sencillo reconocimiento de dichas sustancias y posteriormente se abordan aspectos clave en el abordaje inicial del paciente que ha presentado una exposición de esta índole.
\end{abstract}

PALABRAS CLAVE: intoxicaciones en niños, exposiciones letales en pediatría.

Acta Pediatr Mex. 2016 Jul;37(4):228-240.

\section{CHAMACON: potentially lethal exposures in pediatrics.}

Bazán-Godínez $\mathrm{S}^{1}$, Pérez-Tuñon JG²

\begin{abstract}
Accidental exposure to potentially toxic substances is a common problem in children. This condition could be catastrophic, depending on factors associated with the patient, their environment and the substance involved. In this sense, there is a group of commonly used substances that when ingested by an infant, even in small amounts (one tablet, sip or spoonful), may cause death, either because they are highly toxic or simply because they are in a high concentration. When a child ingests any of these substances, it should be recognized as "a potentially lethal exposure". We propose the mnemonics CHAMA$\mathrm{CON}$ to identify those substances and subsequently, we mentioned key aspects for the initial treatment in this exposures.
\end{abstract}

KEYWORDS: poisoning in children; lethal exposures in pediatrics
${ }^{1}$ Residente de tercer año de la especialidad en pediatría, Hospital Pediátrico Tacubaya, Secretaría de Salud del Distrito Federal.

2Jefe de toxicología hospital Ángeles Lomas. Asistente de la dirección fines de semana y festivos en Hospital Pediátrico Villa.

Recibido: 2 de octubre del 2015

Aceptado: 1 de junio del 2016

\section{Correspondencia}

Jorge Guillermo Pérez Tuñon

Jefe de toxicología hospital Ángeles Lomas

jtunon80@hotmail.com

Este artículo debe citarse como

Bazán-Godínez S, Pérez-Tuñon JG. CHAMACON: exposiciones potencialmente letales en pediatría. Acta Pediatr Mex. 2016;37(4):228-240. 


\section{INTRODUCCIÓN}

La exposición accidental a sustancias potencialmente tóxicas es un problema frecuente en pediatría y particularmente en pacientes que se encuentran en el grupo de entre 1 y 3 años de edad. ${ }^{1}$ En Estados Unidos, en el 2014, la American Association of Poison Control Centers reportó un total de 2,165,142 exposiciones, de las cuales $47.6 \%$ ocurrieron en pacientes de 5 años o menores. ${ }^{2}$ Lo anterior guarda una relación estrecha con factores de riesgo que dependen del paciente, pero también de su entorno y de la propia sustancia, los cuales en su conjunto determinan si se presenta o no una intoxicación. ${ }^{3,4}$

Dentro de los factores predisponentes más importantes del paciente encontramos: su completo desconocimiento del potencial efecto dañino que poseen las sustancias que le rodean y su avidez por la experimentación a través del gusto, además de que su peso y superficie corporal reducidos contribuyen a alcanzar las dosis tóxicas con relativa facilidad. Por otra parte, entre las condiciones ambientales involucradas con estos accidentes podemos observar: la presencia de sustancias tóxicas al alcance de los niños, envases que no cuentan con tapas de seguridad, líquidos tóxicos contenidos en botellas de bebidas o que se presentan en colores vivos, de aspecto o aroma atrayente, así como gavetas o cajones con medicamentos que los familiares conservan en casa. Finalmente, son también importantes las características propias del agente involucrado, entre las que destacan: su sitio de acción, potencia, presentación, concentración y cantidad disponible, las cuales son variables que determinan en conjunto su efecto deletéreo. ${ }^{5,6}$

En ese sentido, existe un grupo de xenobióticos, que no obstante el hecho de que algunos de ellos representan medicamentos con eficacia y seguridad comprobadas, pueden conferir un riesgo elevado de letalidad en pacientes con bajo peso corporal con respecto a la presentación genérica del medicamento o el producto, particularmente en el caso de los pacientes menores de $10 \mathrm{~kg}$, incluso si se trata de lo que sus padres pudieran considerar como "pequeñas cantidades" (una tableta, un sorbo o una cucharada). Lo anterior, en el caso de los medicamentos puede deberse a que poseen un estrecho margen terapéutico o a que su forma farmacéutica está diseñada para administrarse a un paciente adulto, mientras que en el caso de los agentes no terapéuticos puede deberse a que presentan una baja dosis tóxica o a que el producto se encuentra en una elevada concentración. Por ello, cuando un lactante ingiere cualquier sustancia de este grupo debe reconocerse como una "exposición potencialmente letal en pediatría", lo cual implica que el paciente deberá ser hospitalizado para llevar a cabo un monitoreo continuo de sus constantes vitales, así como para aplicar las medidas terapéuticas urgentes que correspondan en cada caso. ${ }^{7-11}$

En el presente trabajo se propone la nemotecnia CHAMACON (Cuadro 1) para tener en consideración a las principales sustancias que, aun ingeridas en pequeñas cantidades, son capaces de causar la muerte a un lactante. Posteriormente se revisan los aspectos básicos del abordaje terapéutico inicial en cada caso; no obstante se recomienda que en todo paciente que presente una exposición potencialmente tóxica se solicite asesoría a un centro de información toxicológica. En el Cuadro 2 se enlistan las presentaciones máximas de estos productos en el mercado mexicano, así como sus dosis letales mínimas.

\section{CLOROQUINA Y ANTIMALÁRICOS}

La cloroquina, hidroxicloroquina, quinina y quinidina son un grupo de medicamentos empleados para el tratamiento del paludismo en zonas endémicas, además de estar indicados en el control de padecimientos reumáticos y como 
Cuadro 1. Ingestas potencialmente letales en pediatría

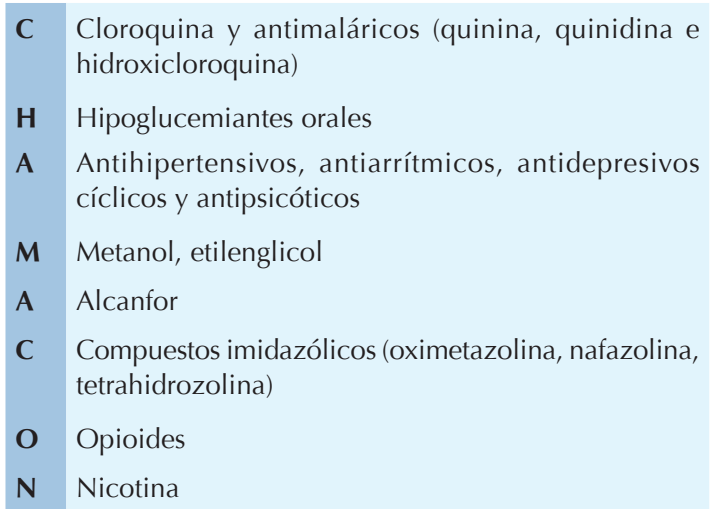

antiarrítmicos debido a su efecto bloqueador de canales iónicos de sodio y potasio (clase IA). Este grupo representa el principal mecanismo por el cual se produce toxicidad en sobredosis, debido a que el bloqueo de los canales rápidos de sodio retrasa la fase 0 del potencial de acción miocárdico y con ello se enlentece la despolarización. Luego de una exposición oral las manifestaciones clínicas tienden a presentarse en las primeras 2 horas, dependiendo de la dosis recibida, y consisten en hipotensión arterial, arritmias (bradicardia sinusal, bloqueo aurículoventricular, taquicardia ventricular, fibrilación, torsades de pointes y asistolia), depresión del estado de alerta, coma, crisis convulsivas y apnea. A su vez, pueden estar presentes síntomas gastrointestinales como vómito, diarrea y cólicos abdominales. Otras alteraciones comprenden hiperkalemia, hipoglucemia, ceguera, ototoxicidad y miotoxicidad. ${ }^{12}$

El diagnóstico se basa en el interrogatorio y los hallazgos clínicos, debido a que las pruebas específicas para estos agentes se encuentran poco disponibles en el medio hospitalario. Las manifestaciones gastrointestinales y la hipotensión suelen ser más acentuadas con quinina y quinidina que con cloroquina e hidroxicloroquina, lo cual puede contribuir al diagnóstico definitivo. El abordaje inicial, al igual que sucede
Cuadro 2. Dosis letal mínima y presentación de fármacos potencialmente letales en pediatría

\begin{tabular}{|c|c|c|}
\hline Fármaco & $\begin{array}{l}\text { Dosis letal } \\
\text { mínima } \\
(\mathrm{mg} / \mathrm{kg})\end{array}$ & $\begin{array}{l}\text { Dosis máxima } \\
\text { disponible* }\end{array}$ \\
\hline $\begin{array}{l}\text { Antimaláricos } \\
\text { Cloroquina } \\
\text { Hidroxicloroquina } \\
\text { Quinina }\end{array}$ & $\begin{array}{l}20 \\
20 \\
80\end{array}$ & $\begin{array}{l}150 \mathrm{mg} \\
200 \mathrm{mg} \\
300 \mathrm{mg}\end{array}$ \\
\hline $\begin{array}{l}\text { Hipog/ucemiantes orales } \\
\text { Glibenclamida } \\
\text { Glipizida }\end{array}$ & $\begin{array}{l}<1 \\
<1\end{array}$ & $\begin{array}{l}5 \mathrm{mg} \\
5 \mathrm{mg}\end{array}$ \\
\hline $\begin{array}{l}\text { Antiarrítmicos y antihiper- } \\
\text { tensivos } \\
\text { Nifedipina } \\
\text { Verapamil } \\
\text { Diltiazem } \\
\text { Disopiramida } \\
\text { Propranolol }\end{array}$ & $\begin{array}{l}15 \\
15 \\
15 \\
15 \\
20\end{array}$ & $\begin{array}{c}60 \mathrm{mg} \\
240 \mathrm{mg} \\
360 \mathrm{mg} \\
150 \mathrm{mg} \\
80 \mathrm{mg}\end{array}$ \\
\hline $\begin{array}{l}\text { Antidepresivos cíclicos } \\
\text { Amitriptilina } \\
\text { Imipramina }\end{array}$ & $\begin{array}{l}15 \\
15\end{array}$ & $\begin{array}{l}50 \mathrm{mg} \\
25 \mathrm{mg}\end{array}$ \\
\hline $\begin{array}{l}\text { Antipsicóticos } \\
\text { Clorpromazina } \\
\text { Clozapina }\end{array}$ & $\begin{array}{l}15 \\
10\end{array}$ & $\begin{array}{l}100 \mathrm{mg} \mathrm{o} \\
10 \mathrm{mg} / \mathrm{mL} \\
200 \mathrm{mg}\end{array}$ \\
\hline $\begin{array}{l}\text { Alcoholes } \\
\text { Metanol } \\
\text { Etilenglicol }\end{array}$ & $\begin{array}{l}500 \\
800\end{array}$ & $\begin{array}{l}\text { Variable } \\
\text { Variable }\end{array}$ \\
\hline $\begin{array}{l}\text { Compuestos imidazólicos } \\
\text { Oximetazolina } \\
\text { Nafazolina }\end{array}$ & $\begin{array}{l}0.1 \\
0.1\end{array}$ & $\begin{array}{c}0.5 \mathrm{mg} / \mathrm{mL} \\
1 \mathrm{mg} / \mathrm{mL}\end{array}$ \\
\hline $\begin{array}{l}\text { Opioides } \\
\text { Codeína } \\
\text { Metadona } \\
\text { Morfina }\end{array}$ & $\begin{array}{l}7 \\
5 \\
1\end{array}$ & $\begin{array}{c}60 \mathrm{mg} \\
40 \mathrm{mg} \\
200 \mathrm{mg}\end{array}$ \\
\hline $\begin{array}{l}\text { Otros } \\
\text { Alcanfor } \\
\text { Nicotina (líquida) }\end{array}$ & $\begin{array}{l}100 \\
0.8\end{array}$ & $\begin{array}{c}200 \mathrm{mg} / \mathrm{mL} \\
24 \mathrm{mg} / \mathrm{mL}\end{array}$ \\
\hline
\end{tabular}

*Basada en la forma farmacéutica de productos comercializados en México.

en otras intoxicaciones, debe incluir las medidas de soporte vital básico y avanzado en caso de requerirse. A la par, se iniciará el monitoreo hemodinámico, se solicitará un electrocardiograma y se llevará a cabo la restitución hídrica necesa- 
ria. La descontaminación gástrica está indicada si se trata de una ingesta reciente, recordando que en pacientes con deterioro del estado de alerta deberá brindarse protección previa de la vía aérea mediante intubación endotraqueal. ${ }^{13,14}$ La aplicación de carbón activado es de utilidad en dosis única (para descontaminación gástrica) y como diálisis gastrointestinal (para inhibir la circulación entero-hepática y entero-entérica). ${ }^{15}$ La hipotensión arterial debe tratarse inicialmente con cristaloides y de ser necesario con aminas vasopresoras. Por otro lado, el ritmo cardiaco debe monitorizarse en forma continua, ya que la longitud del complejo QRS refleja la funcionalidad de los canales de sodio en el miocardio $y$, a medida que se prolonga, puede representar un factor predictor para el desarrollo de arritmias ventriculares, por lo cual es recomendable que con una longitud superior a $100 \mathrm{~ms}$ se inicie tratamiento con bicarbonato de sodio a razón de $1 \mathrm{mEq} / \mathrm{kg} / \mathrm{h}$ en infusión continua hasta lograr un $\mathrm{pH}$ sérico de entre 7.5 y 7.55 o la normalización del complejo. Mientras que para otros trastornos del ritmo se recomienda seguir las guías de atención propuestas por la American Heart Association. ${ }^{12,16}$ En lo referente a las alteraciones neurológicas son necesarias únicamente las medidas de sostén y el apoyo mecánico ventilatorio en caso de depresión respiratoria. ${ }^{17}$

\section{HIPOGLUCEMIANTES ORALES}

Las sulfonilureas de primera generación, como la clorpropamida o la tolbutamida, y de segunda generación como la glibenclamida o la glipizida son hipoglucemiantes orales empleados en el tratamiento de la diabetes mellitus tipo 2. Actúan a nivel de las células beta del páncreas favoreciendo la liberación de insulina; por tal motivo, las principales manifestaciones que presenta un paciente que ingirió accidentalmente uno de estos medicamentos están asociadas con hipoglucemia, ya sea por neuroglucopenia o por estimulación simpática. Los síntomas asociados con neuroglucopenia suelen prevalecer en el paciente menor de 5 años y consisten en: Ilanto por hambre, hipoactividad, fatiga, somnolencia, incoordinación, alteraciones visuales, coma, hipotermia y crisis convulsivas. Por otro lado, las manifestaciones autonómicas pueden presentarse a cualquier edad y se caracterizan por palidez, palpitaciones, taquicardia, hipertensión, diaforesis, ansiedad, temblores, piloerección, sequedad de boca y náusea. Es importante señalar que las secuelas neurológicas, así como la muerte encefálica y el paro cardiorrespiratorio dependen del nivel alcanzado de hipoglucemia y el tiempo que el paciente se mantiene en este estado, por lo que un retraso en el inicio del tratamiento puede resultar catastrófico. ${ }^{18}$

El diagnóstico se basa en la historia clínica y se complementa con la determinación de la glucemia. El tratamiento no debe demorarse en espera de una glucemia central y dependerá de si el paciente se presenta o no sintomático. En lo que se refiere al paciente asintomático que ha ingerido un hipoglucemiante la prioridad será prevenir su absorción, por lo que está indicado realizar una descontaminación gástrica mediante lavado, así como la administración de una dosis única de carbón activado. ${ }^{13,19,20}$ Por otro lado, si las medidas de descontaminación fracasan o si el paciente se presenta con datos clínicos de hipoglucemia, el tratamiento deberá incluir también la reposición de glucosa, administrando una dosis inicial de rescate de 0.5 a $1 \mathrm{~g} / \mathrm{kg}$ de peso en bolo al $10 \%$ si es un lactante y hasta a $25 \%$ para los niños mayores; es decir, 5 a $10 \mathrm{~mL}$ de solución glucosada a 10\% o 2 a 4 mL de solución glucosada a 25\% (relación 1:1 de dextrosa a 50\% y agua bidestilada) por kilogramo de peso. Las soluciones con glucosa en otras presentaciones no se recomiendan para este fin en los pacientes pediátricos debido a que aportan un elevado volumen u osmolaridad dependiendo de su concentración. Dicha dosis de rescate pretende restablecer la función encefálica y resulta indis- 
pensable para una reanimación cardiopulmonar exitosa; no obstante, es de esperarse que debido a su administración se presente una liberación de insulina a nivel pancreático y ello origine nuevamente hipoglucemia en un efecto de rebote al cabo de pocos minutos. Para contrarrestar este efecto puede emplearse octreótido a dosis de 1 a $2 \mu \mathrm{cg} / \mathrm{kg}$ subcutáneo cada 6 a 12 horas, administrar desde el inicio una infusión continua de glucosa suficiente para mantener la glucemia entre 100 y 150 mg/dL, o ambas cosas. ${ }^{21}$ Para tal efecto no existe una dosis establecida debido a que la respuesta depende básicamente de la dosis de sulfonilurea administrada y las reservas previas de glucosa; sin embargo, los autores sugerimos iniciar con aportes de glucosa-kgminuto de 6 a 8 g para lactantes y de 4 a $6 \mathrm{~g}$ para niños mayores, reajustando el aporte de acuerdo con las necesidades del paciente.

\section{ANTIARRÍTMICOS Y ANTIHIPERTENSIVOS}

Se trata de un grupo amplio de medicamentos que se caracterizan por un elevado riesgo de afección hemodinámica, dentro de los cuales, por la frecuencia con que se prescriben, debemos destacar al grupo de los calcioantagonistas y al de los betabloqueadores. Los calcioantagonistas actúan bloqueando los canales de calcio, que son necesarios para llevar a cabo la contracción muscular, ya sea en músculo liso o cardiaco; su efecto central o periférico a dosis terapéuticas depende de la subfamilia a la que pertenecen (dihidropiridinas, benzotiacepinas o fenilalquilaminas); sin embargo, en dosis tóxicas generalmente la afectación se presenta a ambos niveles. Por otro lado, los betabloqueadores actúan de forma competitiva, bloqueando los receptores adrenérgicos beta con lo que disminuyen la producción de AMP cíclico y se atenúan los efectos de las catecolaminas.

Ambos grupos están indicados en padecimientos como la hipertensión arterial, arritmias, insuficiencia cardiaca y migraña refractaria al tratamiento, además los calcioantagonistas se indican en el tratamiento de padecimientos vasculares distales como el fenómeno de Raynaud, mientras que los betabloqueadores en el tratamiento de tirotoxicosis, glaucoma y ataques de pánico.

Los síntomas con frecuencia aparecen entre 1 y 3 horas después de la exposición; sin embargo, actualmente una gran parte de estos medicamentos se comercializan en presentaciones de liberación prolongada, por lo que el paciente puede presentarse, de manera inicial, sin datos clínicos de intoxicación y después evolucionar de forma rápida a la inestabilidad hemodinámica. En este sentido, en ambos grupos predomina la hipotensión arterial, que suele ser severa y refractaria al tratamiento con cristaloides; la bradicardia es otro signo frecuente, aunque con algunos calcioantagonistas inicialmente puede observarse una taquicardia compensatoria a la vasodilatación periférica. Los trastornos de la conducción miocárdica se presentan como bloqueos aurículo-ventriculares, prolongación de QRS, del QT, asistolia, depresión del segmento ST, inversión de onda P y ondas $U$ prominentes. Por otra parte, el deterioro del estado de alerta, las crisis convulsivas y la depresión respiratoria son propias de los calcioantagonistas y el propranolol. Finalmente, los pacientes intoxicados por calcioantagonistas pueden cursar con episodios de hiperglucemia debido al bloqueo de la liberación de insulina, la cual es calcio-dependiente; mientras que los intoxicados por betabloqueadores tienden a desarrollar hipoglucemia debido a la inhibición de la gluconeogénesis y la glucogenólisis hepáticas. ${ }^{22-25}$

El diagnóstico nuevamente debe basarse en los antecedentes y la presentación clínica, siendo la glucemia una herramienta útil para diferenciar entre ambos grupos de medicamentos. Las medidas tempranas de descontaminación como el lavado gástrico y la administración de carbón 
activado en dosis única están indicadas. ${ }^{19,20}$ Para las presentaciones de liberación prolongada puede llevarse a cabo una irrigación intestinal total con polietilenglicol a dosis de $25 \mathrm{a} 40 \mathrm{~mL} /$ $\mathrm{kg} / \mathrm{h}$ hasta obtener un efluente claro, a menos que existan datos de íleo o hipotensión arterial marcada, lo cual contraindica dicho procedimiento. ${ }^{26}$ En forma paralela debe iniciarse el monitoreo electrocardiográfico continuo y la protección de la vía aérea si existe deterioro del estado de alerta o hipotensión arterial sostenida. Si es el caso, se debe iniciar la administración de cristaloides seguida de aminas vasoactivas con la finalidad de mantener una tensión arterial perfusoria. Para tal efecto, la terapia con insulina-glucosa es efectiva, logrando incrementar el inotropismo al incrementar la liberación de catecolaminas y favorecer la permeabilidad de los canales iónicos, además de que la glucosa provee el sustrato energético requerido. Para ello, se administra una infusión continua de glucosa de 0.5 a $1 \mathrm{~g} / \mathrm{kg} / \mathrm{h}$ e insulina de $0.5 \mathrm{a}$ $1 \mathrm{Ul} / \mathrm{kg} / \mathrm{h}$, debiendo mantener la glucemia por arriba de 100 mg/dL. Por otro lado, la emulsión lipídica es un método que incrementa la eliminación de xenobióticos lipofílicos, además de tener un efecto inotrópico positivo; para ello se emplean lípidos a $20 \%$ en un bolo inicial de $1 \mathrm{~mL} / \mathrm{kg}$, seguido de una infusión continua a $15 \mathrm{~mL} / \mathrm{kg} / \mathrm{h}$. En caso de que el paciente se presente en paro cardiorrespiratorio se deberá administrar el bolo inicial durante la secuencia de reanimación cardiopulmonar, siguiendo los protocolos propuestos por la American Heart Association. Por su parte, el calcio representa la primera línea de tratamiento en la intoxicación por calcioantagonistas a dosis de 10 a $20 \mathrm{mg} / \mathrm{kg}$ (0.5-1 mEq/kg) cada 5 a 10 minutos hasta obtener respuesta; ${ }^{27}$ ello se logra con la administración de cloruro de calcio a dosis de 0.4 a $0.7 \mathrm{~mL} / \mathrm{kg}$ (26.8 $\mathrm{mg} / \mathrm{mL})$ o de gluconato de calcio a dosis de 1.1 a $2.3 \mathrm{~mL} / \mathrm{kg}(8.6 \mathrm{mg} / \mathrm{mL})$ para administrarse en 5 a 10 minutos y preferentemente por una vía central. Una alternativa a los bolos es la infusión continua de calcio de 1 a $2.5 \mathrm{mEq} /$ $\mathrm{kg} / \mathrm{h}\left(20\right.$ a $50 \mathrm{mg} / \mathrm{kg}$ ) hasta obtener respuesta. ${ }^{28}$ La L-carnitina en la intoxicación por calcioantagonistas actúa disminuyendo la resistencia a la insulina, promoviendo el transporte intracelular de glucosa, facilitando el metabolismo de ácidos grasos e incrementando la sensibilidad de los canales de calcio, por lo cual se ha sugerido su empleo como terapia adyuvante..$^{29}$ Finalmente, el glucagón es el medicamento de elección para la intoxicación por betabloqueadores con una dosis inicial de $50 \mu \mathrm{g} / \mathrm{kg}$, seguida de una infusión continua de 50 a $100 \mu \mathrm{g} / \mathrm{kg} / \mathrm{h} .{ }^{24,30}$

\section{ANTIDEPRESIVOS CÍCLICOS}

Se trata de un grupo farmacológico que comprende a los antidepresivos tricíclicos y tetracíclicos como amitriptilina, imipramina y amoxapina, entre los más comunes. Estos medicamentos son empleados en el tratamiento de la depresión, dolor neurálgico, migraña, enuresis, trastorno por déficit de atención con hiperactividad y trastorno obsesivo compulsivo, principalmente. Su mecanismo de acción involucra el bloqueo de la recaptura de norepinefrina, dopamina y serotonina en las terminales nerviosas, además de que poseen en grado variable inhibición muscarínica. Por ello, las manifestaciones iniciales pueden ir desde una ligera somnolencia hasta un síndrome anticolinérgico florido, caracterizado por midriasis, taquicardia, piel seca y roja, disminución del peristaltismo, retención urinaria, mucosas secas e hipertermia. La tensión arterial de manera inicial puede encontrarse con tendencia a la elevación; sin embargo se deben esperar estados de hipotensión sostenida, siendo esta condición la causa más frecuente de muerte. Además pueden presentarse arritmias ventriculares, somnolencia, coma y crisis convulsivas. ${ }^{31}$

El diagnóstico se sustentará en la historia clínica, aunque también puede ser de utilidad un perfil toxicológico en orina con un resultado positivo 
a antidepresivos cíclicos. El tratamiento debe incluir las medidas básicas de sostén, así como el monitoreo de la longitud del complejo QRS, el cual a medida que se prolonga guarda una relación directa con complicaciones severas como la aparición de arritmias ventriculares y crisis convulsivas. La descontaminación gástrica debe realizarse aun de forma tardía debido a que, como manifestación de un síndrome anticolinérgico, puede encontrarse retrasado el vaciamiento gástrico; así mismo, la aplicación de carbón activado está indicada en dosis única y en gastrodiálisis siempre que se mantenga conservado el tránsito intestinal. ${ }^{15,19}$ La hipotensión arterial se trata mediante la administración de cristaloides seguida de aminas vasoactivas si así se requiere. ${ }^{32}$ Por su parte, las crisis convulsivas tienden a autolimitarse y se pueden tratar con benzodiacepinas a dosis habituales. El bicarbonato de sodio está indicado si existe alargamiento del complejo QRS (mayor a $100 \mathrm{~ms}$ ), administrando un bolo inicial de 1 a $2 \mathrm{mEq} / \mathrm{kg}$ de peso, seguido de una dosis de mantenimiento en infusión continua de 1 a $2 \mathrm{mEq} / \mathrm{kg} / \mathrm{h}$ hasta lograr el acortamiento de dicho complejo sin elevar el $\mathrm{pH}$ sérico por arriba de 7.55. El antiarrítmico de elección en los casos que no responden al bicarbonato de sodio es la lidocaína en dosis de 1 a $1.5 \mathrm{mg} / \mathrm{kg}$ en bolo lento (2 a 3 minutos), con dosis subsecuentes de 0.5 a $0.75 \mathrm{mg} / \mathrm{kg}$ cada 5 a 10 minutos sin exceder $3 \mathrm{mg} / \mathrm{kg}$ la dosis total. La infusión lipídica puede ser otra alternativa en caso de registrarse falta de respuesta al tratamiento inicial; para lo cual se utilizan lípidos a $20 \%$ en un bolo inicial de $1 \mathrm{~mL} / \mathrm{kg}$, seguido de una infusión continua a $15 \mathrm{~mL} / \mathrm{kg} / \mathrm{h}$. Al igual que en el caso anterior, si el paciente se presenta en paro cardiorrespiratorio, se deberá administrar el bolo inicial durante la secuencia de reanimación cardiopulmonar. ${ }^{33,34}$

\section{ANTIPSICÓTICOS}

Este representa otro grupo nutrido de medicamentos que se clasifican como típicos si su acción depende únicamente del bloqueo de receptores dopaminérgicos, o como atípicos si su efecto se extiende a receptores de 5-hidroxitriptamina, muscarínicos o histaminérgicos. Están indicados en el tratamiento de la esquizofrenia y como adyuvantes en el tratamiento de la depresión, trastornos de la alimentación, insomnio, estrés postraumático y trastorno por déficit de atención con hiperactividad. Por su mecanismo de acción las manifestaciones clínicas son similares a las que se presentan con los antidepresivos cíclicos, siendo posible integrar un síndrome anticolinérgico acompañado de hipotensión arterial, alargamiento del complejo QRS, arritmias ventriculares, somnolencia, coma y crisis convulsivas. Además de ello pueden observarse sedación, coma, depresión respiratoria y síntomas extrapiramidales (distonía y parkinsonismo principalmente). El síndrome neuroléptico maligno es una complicación de aparición tardía (2 a 10 días) y se caracteriza por presentar alteración del estado mental, rigidez muscular, hipertermia e inestabilidad autonómica (fluctuaciones en la frecuencia cardiaca y la tensión arterial). ${ }^{35}$

El diagnóstico se basa en los hallazgos clínicos. El tratamiento consiste en administrar las medidas de sostén. Así mismo, al igual que en los antidepresivos cíclicos, debe monitorizarse la longitud del complejo QRS y de encontrarse mayor a $100 \mathrm{~ms}$ iniciar terapia con bicarbonato de sodio. En caso de presentar hipertermia el control de la temperatura con enfriamiento externo es fundamental en la prevención de complicaciones neurológicas. El lavado gástrico y la administración de carbón activado están indicados aún de manera tardía debido al retraso en el vaciamiento gástrico por efecto anticolinérgico, recordando que se debe verificar el peristaltismo antes de administrar el carbón activado. ${ }^{19,20}$ La hipotensión arterial debe tratarse también con la administración de volumen y de ser necesario con aminas vasoactivas. Las ben- 
zodiacepinas son de utilidad en el tratamiento de las crisis convulsivas, así como en el control de los síntomas neurológicos del síndrome neuroléptico maligno. ${ }^{36}$ Finalmente, al tratarse de xenobióticos liposolubles, el empleo de lípidos a 20\% está indicado, al igual que en los grupos anteriores, a través de un bolo inicial de $1 \mathrm{~mL} /$ $\mathrm{kg}$, seguido de una infusión continua a $15 \mathrm{~mL} /$ $\mathrm{kg} / \mathrm{h}$ y administrándose durante la secuencia de reanimación cardiopulmonar en caso de sufrir paro cardiorrespiratorio. ${ }^{34}$

\section{ALCOHOLES}

Se trata de compuestos orgánicos que contienen por lo menos un grupo hidroxilo $(-\mathrm{OH})$ unido a un átomo de carbono saturado. No obstante, todos los alcoholes se consideran tóxicos para el humano. Debemos destacar al metanol y al etilenglicol como los que representan el riesgo más elevado de letalidad. Estos agentes es posible encontrarlos en una gran cantidad de productos de uso común. El metanol se utiliza como adulterante de etanol en bebidas que se adquieren ilegalmente, pero también se emplea como combustible en iniciadores de fuego y como solvente en líquidos para limpiaparabrisas, tintas de fotocopiado y perfumes. En lo que respecta al etilenglicol, con frecuencia constituye el principal ingrediente de los anticongelantes, pero se añade también a los líquidos para frenos e hidráulico y se emplea como disolvente en tintas y compuestos para el revelado, entre otros productos de uso industrial. El efecto tóxico en ambos alcoholes es provocado por sus respectivos metabolitos: ácido fórmico (metanol), ácido glioxílico y ácido oxálico (etilenglicol), los cuales condicionan acidosis metabólica y lesión a órgano blanco. Clínicamente, el cuadro se manifiesta por un periodo de embriaguez inicial, similar a la que se observa con el etanol; sin embargo, a medida que el compuesto es biotransformado se hace evidente la acidosis metabólica. Posteriormente, para el caso del metanol los síntomas como ceguera, alteraciones del equilibrio y parkinsonismo están asociados con la acumulación de su metabolito en retina y ganglios basales, mientras que en la exposición a etilenglicol predomina la falla renal por la formación de cristales de oxalato de calcio, lo cual también puede llevar a hipocalcemia con alargamiento del intervalo QT y arritmias. ${ }^{37-39}$

El diagnóstico nuevamente debe basarse en la presentación clínica, siendo ésta una de las principales causas de acidosis metabólica con brecha aniónica amplia. En este contexto debe evaluarse la correlación que guarda la brecha osmótica (osmolaridad medida-osmolaridad calculada) con la brecha aniónica ( $\mathrm{Na}-[\mathrm{Cl}+\mathrm{HCO} 3])$ ya que cuando predomina la primera significa que existe en sangre una cantidad considerable de alcohol y el tratamiento debe encaminarse a evitar su biotransformación mediante la inhibición de la alcohol deshidrogenasa; por el contrario, cuando se cuenta con una brecha aniónica amplia y una osmótica reducida esto significa que la mayor parte del alcohol ha sido transformado en su metabolito y, por lo tanto, el tratamiento debe centrarse en la eliminación de este último por medio de hemodiálisis. Cabe aclarar que la cuantificación sérica o urinaria de estos alcoholes y sus metabolitos se encuentra poco disponible y no se correlaciona adecuadamente con la clínica. Por otro lado, para el etilenglicol el diagnóstico puede apoyarse en la evidencia de cristales de oxalato de calcio en orina o a través de una prueba de fluorescencia con lámpara de wood. Con respecto al tratamiento, debido a que estos compuestos presentan una rápida absorción, además de una mala adsorción al carbón activado, deberán evitarse tanto el vaciamiento gástrico como la administración de carbón activado. ${ }^{37}$ En la inhibición de la alcohol deshidrogenasa es de elección el fomepizol sobre el etanol, debido a que el primero no induce embriaguez, hipoglucemia o irritación gástrica, que representan problemas frecuentes con el empleo 
de etanol (la irritación gástrica se presenta con la administración enteral de etanol). ${ }^{40,41}$ El fomepizol se indica con una dosis inicial de $15 \mathrm{mg} / \mathrm{kg}$, seguido de dosis de mantenimiento de $10 \mathrm{mg} /$ kg cada 12 horas, reajustándolas a cada 4 horas en caso de que se requiera hemodiálisis. Una alternativa eficaz es el etanol por vía enteral o intravenosa, con una dosis inicial de $800 \mathrm{mg} / \mathrm{kg}$ de peso para administrar en 20 minutos, seguida de dosis de mantenimiento de 80 a $150 \mathrm{mg} / \mathrm{kg}$ por hora, manteniendo al paciente en un estado leve de embriaguez (100 a $150 \mathrm{mg} / \mathrm{dL}$ en suero) ${ }^{37-39}$ en este caso, si se inicia terapia con hemodiálisis el aporte también deberá ajustarse de 250 a $350 \mathrm{mg} / \mathrm{kg}$ por hora. Por otro lado, en ausencia de etanol para uso endovenoso se puede recurrir a un preparado por vía enteral como el vodka, que se encuentra alrededor de $40 \%$, debiendo administrarlo en una concentración de 10 a $20 \%$, la cual se obtiene diluyendo el vodka en una relación 1:2 o 1:3 con solución de glucosa a $5 \%$ y se administra por sonda nasogástrica; con esta dilución se aportan 100 a 200 mg de etanol por cada mililitro, respectivamente. ${ }^{42}$ Para favorecer la tolerancia, es de utilidad el empleo de un antiemético de acción central como el ondansetrón. Con respecto a la hemodiálisis, ésta debe indicarse cuando el paciente presenta datos de insuficiencia renal, acidosis metabólica severa $(\mathrm{pH}<7.1)$, daño a órgano blanco o deterioro clínico a pesar del manejo establecido. Finalmente, la diálisis peritoneal es un método poco efectivo y debe evitarse, a menos que no sea posible trasladar al paciente a una unidad que cuente con hemodiálisis. ${ }^{39}$

\section{COMPUESTOS IMIDAZÓLICOS}

La nafazolina, oximetazolina y tetrahidrozolina son agentes derivados de la piperazina, que por su efecto $\alpha_{1}$-adrenérgico se utilizan como vasoconstrictores en el tratamiento local de procesos inflamatorios, principalmente nasales y oftálmicos. Sin embargo, cuando son ingeridos muestran un efecto agonista en los receptores $\alpha_{2}$ adrenérgicos a nivel central y periférico, por lo que al cabo de unos minutos pueden presentarse: bradicardia, hipotensión, hipotermia, palidez y deterioro del estado de alerta con depresión respiratoria. En algunos casos el cuadro va precedido de un breve periodo de hipertensión arterial por estímulo $\alpha_{1} \cdot{ }^{43}$

Debido a que no existen pruebas específicas el diagnóstico se basa en la presentación clínica. En este caso la descontaminación gástrica posee un papel muy limitado debido a la rápida absorción de estos compuestos, por lo que el tratamiento se basa en la terapia de sostén, la cual consiste en administración de cristaloides como primera medida para corregir los estados hipotensivos, seguida del apoyo con aminas en caso de requerirse. Si el paciente se presenta con deterioro del estado de alerta debe asegurarse oportunamente la vía aérea debido al riesgo de depresión respiratoria. Las manifestaciones tienden a desaparecer en un periodo de 8 a 24 horas aproximadamente. ${ }^{43-45}$

\section{OPIOIDES}

Los opioides constituyen un grupo de fármacos con afinidad selectiva por los receptores del mismo nombre, los cuales son empleados para el control del dolor moderado a severo, como antitusivos, antidiarreicos e inductores anestésicos. De igual forma, debido a su efecto euforizante algunos opioides se han utilizado como sustancias de abuso, generando fenómenos de dependencia, tolerancia y síndromes de abstinencia en sus consumidores. Los efectos a dosis terapéuticas dependen de su afinidad por los diferentes receptores (MOP, KOP, DOP y NOP); no obstante, en sobredosis dicha afinidad tiende a desaparecer provocando un síndrome opioide florido, el cual se caracteriza por la triada de: depresión del estado de alerta, miosis y depresión respiratoria. Adicionalmente pueden 
haber íleo, náusea, vómito y alucinaciones. ${ }^{46}$ Con algunos agentes específicos pueden presentarse crisis convulsivas (morfina, tramadol, propoxifeno y meperidina) y trastornos del ritmo cardiaco (propoxifeno). ${ }^{47}$

Además de la integración de un síndrome opioide el diagnóstico puede apoyarse en la determinación de un perfil toxicológico en orina positivo a opioides o a agentes específicos (morfina, heroína, metadona). El tratamiento para un paciente que se presenta sin depresión respiratoria podría limitarse a la descontaminación gástrica, recordando que los opioides en su forma sólida pueden encontrarse tardíamente en el estómago debido a que inhiben el tránsito gastrointestinal, por lo que puede llevarse a cabo un lavado gástrico aún varias horas después de una exposición a opioides en presentaciones sólidas. Por el contrario, la administración de carbón activado podría contraindicarse si se presenta íleo como consecuencia del efecto citado. ${ }^{19,20}$ En los pacientes con depresión de la función ventilatoria el tratamiento se basa en el restablecimiento de la misma, para ello puede ser necesario el manejo avanzado de la vía aérea, así como la administración de un antagonista opioide, siempre que no exista antecedente de uso crónico de estos agentes debido al riesgo de desencadenar un síndrome de abstinencia.

Los antagonistas opioides tampoco deben administrarse en condiciones de hipoxia, por su asociación con edema pulmonar agudo. El antagonista de elección es la naloxona y debe ser administrada la dosis mínima necesaria para restablecer la función ventilatoria, aunque el paciente se mantenga bajo sedación. Para ello se indica una dosis inicial de $0.04 \mathrm{mg} I \mathrm{~V}$, incrementando a $0.4 \mathrm{mg}, 2 \mathrm{mg}$ y $10 \mathrm{mg}$ cada 2 a 5 minutos si no existe respuesta, recordando que debido a la vida media de la naloxona (40 a 60 minutos) puede ser necesario repetir las dosis o administrar en infusión continua si se trata de un opioide de vida media prolongada. En caso de presentarse con crisis convulsivas el tratamiento consiste en la administración de benzodiacepinas hasta obtener respuesta. ${ }^{46,48,49}$

\section{ALCANFOR}

Es un terpenoide que originalmente se obtenía del árbol alcanforero (Cinnamomum camphora) y que en la actualidad es sintetizado a partir de trementina. Son muchas las propiedades que se le atribuyen. En medicina se emplea para el alivio de dolores musculares, óseos o articulares, para el alivio de la constipación nasal, como antipruriginoso, antiséptico o repelente de insectos; a su vez, en la medicina alternativa se suele aplicar sobre las mucosas o por vía oral como antitusígeno, afrodisíaco, anticonceptivo, abortifaciente, supresor de la lactancia y neuroestimulante. Se piensa que su efecto analgésico, al igual que el neurotóxico, puede estar mediado por un agonismo potente e irreversible de los receptores de potencial transitorio vaniloides tipo 1 (TRPV1). Después de la exposición los síntomas tardan en presentarse entre 5 y 90 minutos, manteniéndose por un periodo aproximado de 24 horas. Son comunes odinofagia, náusea, vómito y dolor abdominal por efecto irritativo; posteriormente hay sensación de calor, ansiedad, sedación, debilidad, cefalea, vértigo, mioclonías e hiperreflexia. Los casos severos cursan con desorientación, alucinaciones y crisis convulsivas, aunque éstas últimas pueden ser la primera y única manifestación de la intoxicación e incluso presentarse como un estado epiléptico. El característico olor a acre suele ser evidente en el aliento, el contenido gástrico y la orina. Cabe aclarar que a pesar de que también se pueden presentar datos de falla hepática y depresión respiratoria, la principal causa de muerte es el edema cerebral relacionado con la persistencia de las crisis convulsivas. ${ }^{50}$

El diagnóstico debe ser clínico y el tratamiento estar encaminado a las medidas de soporte, 
incluyendo el control de las crisis convulsivas mediante el empleo de benzodiacepinas hasta obtener respuesta, la inducción de coma barbitúrico puede ser necesaria en algunos casos. Por otra parte, debido a que presenta una rápida absorción, el lavado gástrico se reservará sólo para casos en los que se ingirió una gran cantidad de alcanfor de manera reciente. El carbón activado no ha mostrado efectividad en este caso. ${ }^{51-53}$

\section{NICOTINA}

La nicotina es un alcaloide obtenido de la planta Nicotiana tabacum, el cual representa una de las sustancias de abuso de mayor distribución a nivel mundial. Además de estar presente en puros, cigarros y en estado líquido para uso recreativo, también se le encuentra como tratamiento sustitutivo de la dependencia a nicotina en goma de mascar y parches. Los accidentes por ingesta de colillas de cigarro (0.5-1 mg por pieza) o goma de mascar (2 mg por pieza) son poco frecuentes; sin embargo, con el advenimiento de las presentaciones líquidas que se emplean en los cigarros electrónicos y debido a su elevada concentración, es que las intoxicaciones letales por nicotina tienden a incrementarse de manera alarmante. ${ }^{54}$ Este alcaloide es un potente agonista de los receptores nicotínicos de acetilcolina, por lo que las manifestaciones clínicas son de inicio temprano y consisten en ansiedad, confusión, ataxia, taquicardia, hipertensión, temblor, diarrea, vómito, debilidad, fasciculaciones, crisis convulsivas y arritmias. Con menor frecuencia se presentan datos muscarínicos como salivación, bradicardia o hipotensión. ${ }^{55}$

El diagnóstico debe ser clínico, apoyado en los datos obtenidos en el interrogatorio. En cuanto al tratamiento, no existe un antídoto específico para nicotina, por lo que debe basarse en las medidas de sostén. La descontaminación gástrica mediante lavado y carbón activado es de utilidad en la ingesta de cigarros, colillas o goma de mascar, pero no en presentaciones líquidas debido a la rapidez con que estas son absorbidas. Las benzodiacepinas están indicadas en el tratamiento de la ansiedad y las crisis convulsivas, además de que luego de su administración, la frecuencia cardiaca y la tensión arterial también tienden a disminuir. De no ser así, también puede requerirse el empleo de vasodilatadores; por el contrario, en los casos en que predominan la hipotensión y la bradicardia, éstas pueden tratarse con administración de cristaloides y atropina, respectivamente..$^{55,56}$ Finalmente, los trastornos del ritmo cardiaco se deben tratar con base en los protocolos vigentes de la American Heart Association. ${ }^{16,56}$

\section{CONCLUSIONES}

A pesar de que la mayor parte de las exposiciones ocurren en la edad pediátrica, más de la mitad de los xenobióticos involucrados resultan en un nulo o bajo riesgo para la salud, lo cual se refleja en el bajo porcentaje de letalidad por intoxicaciones en los niños si se le compara con el de los adultos. ${ }^{2}$ Como consecuencia, es una idea común que la ingesta de una pequeña cantidad de cualquier sustancia no será capaz de producir efectos devastadores en nuestro paciente, cuando en realidad existen xenobióticos que pueden causar la muerte aún si se ingiere sólo una tableta, un sorbo o una cucharada de ellos. Lo anterior obliga al médico pediatra a estar familiarizado con las principales sustancias tóxicas y su manejo. No obstante, en México, el pediatra en formación se enfrenta a diversas limitantes entre las que destacan: un programa académico con escasos contenidos en toxicología clínica, la ausencia de libros de texto actualizados en estos temas, la escasez de médicos especialistas en toxicología clínica en las unidades hospitalarias formadoras de residentes y la falta de publicaciones nacionales al respecto. La nemotecnia propuesta en este artículo pretende ser un elemento que contribuya a romper este paradigma. 
Por otro lado, con respecto a la literatura norteamericana y europea, encontramos que las publicaciones en torno al tema generalmente se centran en los xenobióticos más frecuentes de su entorno, aportando limitada información sobre agentes como el alcanfor o los antimaláricos que provocan accidentes con mayor frecuencia en países en desarrollo..$^{1,3,4}$ En lo que respecta a la nicotina, aún se menciona poco dentro del grupo de "exposiciones potencialmente letales en pediatría"; sin embargo, debido a la reciente inclusión de presentaciones líquidas, los accidentes asociados con este agente son cada vez más frecuentes y confieren un riesgo real de letalidad a bajas dosis, ${ }^{54,56}$ por lo que se determinó su inclusión en el presente trabajo.

Por otro lado, una medida efectiva para prevenir cualquier exposición accidental es la educación de pacientes y familiares. En este sentido, conviene recordar a los padres que tanto medicamentos como productos químicos que se encuentran en el hogar deberán encontrarse siempre bajo llave y en su envase original, ya que sólo colocarlos fuera del alcance de los niños no representa una medida eficaz. Así mismo, deberán preferirse aquellos productos con tapas de seguridad, que se encuentren autorizados para su uso en el hogar.

En el caso de los medicamentos, éstos nunca deberán conservarse una vez concluido el esquema de tratamiento, además, siempre deberá revisarse la prescripción en la receta antes de su administración y bajo ninguna circunstancia se le mencionará al niño que "el medicamento es un dulce" o que "sabe rico". ${ }^{57}$ Finalmente, se recomienda al lector consultar el Centro de Información Toxicológica más cercano, el cual puede encontrar en: www.retomex.org.mx.

\section{REFERENCIAS}

1. Shannon M. Ingestion of toxic substances by children. $\mathrm{N}$ Engl J Med. 2000;(342):186-91.
2. Mowry JB, Spyker DA, Brooks DE, et al. 2014 Annual Report of the American Association of Poison Control Centers' National Poison Data System (NPDS): 32st Annual Report. Clin Tox. 2015; 53(10):962-1146.

3. Bryant $\mathrm{S}$, Singer J. Management of toxic exposure in children. Emerg Med Clin N Am. 2003;21:101-19.

4. Abbruzzi G, Stork CM. Pediatric toxicologic concerns. Emerg Med Clin North Am 2002;(20):223-47.

5. Hoffman RS, Howland MA, Levin NA, et al. Goldfrank's Toxicologic emergencies. Mc Graw-Hill. 10a ed. 2015;(32):743-61.

6. Mintegui S. Manual de intoxicaciones en pediatría. 2a edición. Ergon. España. 2008(3):55-117.

7. Liebelt EL, Shannon M. Small doses, big problems: a selected review of highly toxic common medications. Pediatr Emerg Care. 1993;9(5):292-7.

8. Matteucci M. One pill can kill: assessing the potential for fatal poisonings in children. Pediatr Ann. 2005;(34):964-8.

9. Bar-Oz B, Levichek Z. Medications that can be fatal for a toddler with one tablet or teaspoonful: a 2004 update. Paediatr drugs. 2004;6(2):123-6.

10. Braitberg G, Oakley E. Small dose...big poison. Aust Fam Physician. 2010;39(11):826-33.

11. Michael JB, Sztajnkrycer. Deadly pediatric poisons: nine common agents that kill at low doses. Emerg Med Clin North Am. 2004;22(4):1019-50.

12. White NJ. Cardiotoxicity of antimalarial drugs. Lancet Infect Dis. 2007;(7):549-58.

13. Greene S, Harris C, Singer J. Gastrointestinal Decontamination of the Poisoned Patient. Pediatr Emerg Care. 2008;24(3):176-86.

14. Albertson TE, Owen KP, Sutter ME. Gastrointestinal decontamination in the acutely poisoned patient. Int J Em Med. 2011;(4): 65.

15. Position statement and practice guidelines on the use of multi-dose activated charcoal in the treatment of acute poisoning. American Academy of Clinical Toxicology; European Association of Poisons Centres and Clinical Toxicologists. J Toxicol Clin Toxicol.1999;(37):731-51.

16. American Heart Association: 2010 Guidelines for cardiopulmonary resuscitation and emergency cardiovascular care. Part 14: pediatric advanced life support. Circulation. 2010;(122):S876-S908.

17. Yanturali S. Diazepam for treatment of massive chloroquine intoxication. Resuscitation. 2004;(63):347-8.

18. Glatstein M, Garcia-Bournissen F, Scolnik D, et al. Sulfonylurea intoxication at a tertiary care paediatric hospital. Can J Clin Pharmacol. 2010;(17):51-6.

19. Benson BE, Hoppu K, Troutman WG, et al. Position paper update: gastric lavage for gastrointestinal decontamination. Clin Toxicol. 2013;(51):140-6.

20. Chyka PA, Seger D, Krenzelok EP, et al. Position paper: single-dose activated charcoal. Clin Toxicol EUA. 2005;(43):61-87. 
21. Boyle PJ, Justice K, Krentz AJ, et al. Octreotide reverses hyperinsulinemia and prevents hypoglycemia induced by sulfonylurea overdoses. J Clin Endocrinol Metab.1993;(76):752-6.

22. Belson MG, Gorman SE, Sullivan K, et al. Calcium channel blocker ingestions in children. Am J Emerg Med. 2000;(18):581-6.

23. Love JN, Sachdeva DK. Are 1 - 2 tablets dangerous? Beta blocker exposure in toddlers. J Emerg Med. 2004;(26):309-14.

24. DeWitt C, Waksman J. Pharmacology, pathophysiology and management of calcium channel blocker and beta blocker toxicity. Toxicol Rev. 2004;(23):223-38.

25. St-Onge M, Dubé PA, Gosselin S, et al. Treatment for calcium channel blocker poisoning: a systematic review. Clin Toxicol (Phila). 2014;52(9):926-44.

26. American Academy of Clinical Toxicology, European Association of Poison Centers and Clinical Toxicologists. Position Paper: Whole Bowel Irrigation. Clin Toxicol 2004;42(6):843-54.

27. Salhanick SD, Shannon MW. Management of calcium channel antagonist overdose. Drug Saf. 2003;(26):65-79.

28. Broner CW, Stidham GL, Westenkirchner DF, et al. A prospective, randomized, double-blind comparison of calcium chloride and calcium gluconate therapies forhypocalcemia in critically ill children. J Pediatr. 1990;(117):986-9.

29. St-Onge $\mathrm{M}$, Ajmo I, Poirier $\mathrm{D}$, et al. L-Carnitine for the treatment of a calcium channel blocker and metformin poisoning. J Med Toxicol. 2013;9(3):266-9.

30. Auzépy $P$, Boukara N, Richard C, et al. Acute poisoning caused by beta blockers in the adult. Apropos of 7 cases. Ann CardiolAngeiol. 1983;32(4):253-8.

31. Rosenbaum TG, Kou M. Are one or two dangerous? Tricyclic antidepressant exposure in toddlers. J Emerg Med. 2005;(28):169-74.

32. Shannon MW, Merola J, Lovejoy Jr FH. Hypotension in severe tricyclic antidepressant overdose. Am J Emerg Med. 1988;(6):439-42.

33. Sholten HA, Nap A, Bouwman RA, et al. Intralipid as an antidote for tricyclic antidepressants and SSARIs: a case report. Anaesth Intensive Care. 2012;(40):1076-7.

34. Cao D, Heard K, Foran M, et al. Intravenous lipid emulsion in the emergency department: a systematic review of recent literature. J Emerg Med. 2015;48(3):387-97.

35. Bhanushali MJ, Tuite PJ. The evaluation and management of patients with neuroleptic malignant syndrome. Neurol Clin. 2004;(22):389-411.

36. Minns AB, Clark RF. Toxicology and overdose of atypical antipsychotics. J Emerg Med. 2012;(43):906-13.

37. Jacobsen D, McMartin KE. Methanol and ethylene glycol poisonings. Mechanism of toxicity, clinical course, diagnosis and treatment. Med Toxicol. 1986;1(5):309-34.

38. Sivilotti MLA. Ethanol: tastes great! Fomepizole: less filling! Ann Emerg Med. 2008;(53):451-3.
39. Barceloux DG, Bond GR, Krenzelok EP, et al. American Academy of Clinical Toxicology practice guidelines on the treatment of methanol poisoning. J Toxicol Clin Toxicol. 2002;(40):415-46.

40. Beatty L,Green R, Magee K, et al. A systematic review of ethanol and fomepizole use in toxic alcohol ingestions. Emerg Med Int. 2013;(2013):1-14.

41. Brent J. Fomepizole for the treatment of pediatric ethylene and diethylene glycol, butoxyethanol and methanol poisonings. Clin Toxicol (Phila). 2010;48(5):401-6.

42. Roldán J, Frauca C, Dueñas A. Intoxicación por alcoholes. ANALES Sis San Navarra. 2003;26(1):129-39.

43. Mahieu LM, Rooman RP, Goossens E.Imidazoline intoxication in children. Eur J Pediatr. 1993;152(11):944-6.

44. Bucaretchi F, Dragosavac S, Vieira RJ. Acute exposure to imidazoline derivatives in children. J Pediatr (Rio J). 2003;79(6):519-24.

45. Higgins GL, Campbell B, Wallace K, et al. Pediatric poisoning from over-the-counter imidazoline-containing products. Ann Emerg Med. 1991;20(6):655-8.

46. Boyer EW. Management of Opioid Analgesic Overdose. N Engl J Med. 2012;367(2):146-55.

47. McCarron MM, Challoner KR, Thompson GA. Diphenoxylate-atropine (Lomotil) overdose in children: an update (report of eight cases and review of the literature). Pediatrics. 1991;(87):694-700.

48. Sachdeva DK, Stadnyk JM. Are one or two dangerous? Opioid exposure in toddlers. J Emerg Med 2005;(29):77-84.

49. Gonzalez JP, Brogden RN. Naltrexone: A review of its pharmacodynamic and pharmacokinetic properties and therapeutic efficacy in the management of opioid dependence. Drugs. 1988;(35):192-213.

50. American Academy of Pediatrics Committee on Drugs. Camphor revisited: focus on toxicity. Pediatrics. 1994;(94):127-8.

51. Patra C, Sarkar S, Dasgupta MK, et al. Camphor poisoning: An unusual cause of seizure in children. J Pediatr Neurosci. 2015;10(1):78-9.

52. Emery D, Corban J. Camphor toxicity. J Paediatr Child Health. 1999;(35):105-6.

53. Love JN, Sammon M, Smereck J. Are one or two dangerous? Camphor exposure in toddlers. J Emerg Med. 2004;(27):49-54.

54. Appleton S. Frequency and outcomes of accidental ingestion of tobacco products in young children. Reg Tox Pharmacol. 2011;(61):210-4.

55. Bonadio WA, Anderson Y. Tobacco ingestions in children. Clin Pediatr. 1989;(28):592-3.

56. Connolly GN, Richter P, Aleguas A, et al. Unintentional child poisonings through ingestion of conventional and novel tobacco products. Pediatrics. 2010;(125):896-9.

57. Centro para el Control del Envenenamiento de la Ciudad de Nueva York. Publicaciones: Usted puede proteger su casa contra venenos. En: http://poisoncontrol.vanguarddirect. com/es. Acceso 06 de enero de 2016. 